\title{
Topological invariants and the definition of energy in quadratic gravity theory
}

\author{
Gaston Giribet \\ Physics Department, University of Buenos Aires and IFIBA-CONICET, \\ Ciudad Universitaria, pabellón 1, 1428 Buenos Aires, Argentina \\ Olivera Miskovic ${ }^{\dagger}$ \\ Instituto de Física, Pontificia Universidad Católica de Valparaíso, Casilla 4059, Valparaíso, Chile \\ Rodrigo Olea $\oplus^{\ddagger}$ \\ Departamento de Ciencias Físicas, Universidad Andres Bello, Sazié 2212, Piso 7, Santiago, Chile \\ David Rivera-Betancour $\odot^{\S}$ \\ CPHT-Centre de Physique Théorique, CNRS, École Polytechnique, Institut Polytechnique de Paris, \\ Route de Saclay, 91128 Palaiseau, France
}

(Received 31 January 2020; accepted 10 March 2020; published 20 March 2020)

\begin{abstract}
We provide a simple method to compute the energy in higher curvature gravity in asymptotically AdS spacetimes in even dimensions. It follows from the combined use of topological terms added to the gravity action, and the Wald charges derived from the augmented action functional. No additional boundary terms are needed. As a consistency check, we show that the formula for the conserved quantities derived in this way yields the correct result for the mass of asymptotically AdS black holes.
\end{abstract}

DOI: $10.1103 /$ PhysRevD.101.064046

\section{INTRODUCTION}

Higher-curvature corrections to Einstein-Hilbert action are ubiquitous in effective field theory when gravity is involved. In fact, a sensible theory of quantum gravity is generically expected to yield such corrections, and string theory, as an antonomastic example of this, does predict them at next-to-leading order [1-3]. Higher-curvature corrections, on the other hand, are interesting by their own right: they have been studied for a long time and in many different contexts, including mathematical aspects of general relativity (GR) [4-6], cosmology [7], black holes [8], massive gravity [9], supergravity [10], and quantum gravity [11].

Regarding quantum gravity, it is well known that the introduction of higher-order terms in the gravity action suffices to render the theory renormalizable [11] but at the

\footnotetext{
gaston@df.uba.ar

olivera.miskovic@pucv.cl

*odrigo.olea@unab.cl

§david.rivera-betancour@zimbra.polytechnique.fr
}

Published by the American Physical Society under the terms of the Creative Commons Attribution 4.0 International license. Further distribution of this work must maintain attribution to the author(s) and the published article's title, journal citation, and DOI. Funded by SCOAP ${ }^{3}$. price of introducing ghosts [9]. This is generically the case, with a few notable exceptions [3]. Therefore, the absence of ghosts and of other potential pathologies, such as causality issues, can be used as a criterion to select the sensible higher-order theory or ultraviolet completion to work with $[3,12,13]$.

In recent years, the interest in healthy higher-order corrections to Einstein theory has been renewed. In particular, there have been very interesting works studying higher-curvature models in anti-de Sitter (AdS) space. Such is the case of the so-called critical gravity (CG) theories [14,15], which provide ghost-free models of gravity in asymptotically AdS spacetimes in $D \geq 4$ dimensions. In $D=3$ dimensions, higher-curvature terms were also considered as a toy model of a consistent gravity theory [16]. Other recent works explore, for instance, the connection between higher-curvature conformally invariant theories and Einstein gravity in (A)dS in $D=4$ [17]. Other models in $D \geq 4$ dimensions studied recently, such as the socalled (generalized) quasitopological gravity [18-23] and Einstenian gravity [24,25], are also very interesting and provide a new perspective on this old topic.

The computation of conserved charges in highercurvature theories in both asymptotically flat and asymptotically AdS spaces is an important problem that has been addressed by many authors in the last 20 years; notably by 
Deser and Tekin, who found in Refs. [26,27] the higherderivative generalization of the Abbot-Deser method for GR [28]. Other papers discussing conserved charges in related contexts are [29-31], and of course others using the covariant formalism of Ref. [32]; see references therein.

Recently, we proposed in [33] a novel definition of gravitational energy for an arbitrary theory of gravity including quadratic-curvature corrections to Einstein equations. We focused on the theory in $D=4$ dimensions and in the presence of a negative cosmological constant. Unlike some other methods considered in the literature, the method proposed in [33] is intrinsically nonlinear and permits one to deal with the boundary terms $[34,35]$ in a systematic way. It relies on the idea of adding to the gravity action topological invariant terms, which suffice to regularize the Noether charges and render the variational problem well posed. More precisely, the method amounts to adding to the action the bulk part of a topological invariant. This is an idea that has been previously considered in $[36,37]$ in the case of second-order theories, such as Einstein-AdS gravity. Here, we show how this method can be generalized to generic quadratic-curvature theories in any even dimension.

The paper is organized as follows: In Sec. II, we will introduce the action of generic quadratic-curvature gravity in $D=2 n$ dimensions, including topological invariants that will eventually serve to regularize the action. In Sec. III, we provide the definition of gravitational energy and we use it to compute the mass of black holes in asymptotically $\operatorname{AdS}_{D}$ space. Section IV contains our conclusions.

\section{QUADRATIC-CURVATURE GRAVITY IN $D=2 n$ DIMENSIONS}

The most general gravity action which adds up quadratic corrections in the curvature to GR in even dimensions is given by the expression

$$
\begin{aligned}
I= & \int_{M} d^{2 n} x \sqrt{-g}\left(\frac{R-2 \Lambda_{0}}{\kappa}+\alpha R_{\nu}^{\mu} R_{\mu}^{\nu}+\beta R^{2}+\gamma G B\right) \\
& +\alpha_{2 n} \int_{M} d^{2 n} x \mathcal{E}_{2 n} .
\end{aligned}
$$

Here, $G B \equiv \sqrt{-g}\left(R^{\alpha \beta \mu \nu} R_{\alpha \beta \mu \nu}-4 R^{\mu \nu} R_{\mu \nu}+R^{2}\right)$ is the GaussBonnet term, which in $D>4$ is a dynamical term. The last term in the integral (1) is, by contrast, the $2 n$-dimensional topological Euler density, which reads

$$
\mathcal{E}_{2 n}=\frac{\sqrt{-g}}{2^{n}} \delta_{\left[\nu_{1} \cdots \nu_{2 n}\right]}^{\left[\mu_{1} \cdots \mu_{2 n}\right]} R_{\mu_{1} \mu_{2}}^{\nu_{1} \nu_{2}} \cdots R_{\mu_{2 n-1} \mu_{2 n}}^{\nu_{2 n-1} \nu_{2 n}}
$$

This contribution accommodates the maximal number of curvatures in $2 n$ dimension, such that it does not contribute to the field equations. In $D=4$, this invariant is the same as the Gauss-Bonnet term.
We shall use the conventions in Ref. [27], where $\kappa=$ $2 \operatorname{Vol}\left(S^{2 n-2}\right) G_{2 n}$ in terms of the $D$-dimensional Newton's constant $G_{D}$. In addition, in order to compare with existing literature, we are considering the inclusion of a bare cosmological constant $\Lambda_{0}=-(2 n-1)(2 n-2) / 2 \ell^{2}$ in terms of the original AdS radius $\ell$. In our notation, $\delta_{\left[\nu_{1} \cdots \nu_{p}\right]}^{\left[\mu_{1} \cdots \mu_{p}\right]}=\operatorname{det}\left[\delta_{\nu_{1}}^{\mu_{1}} \cdots \delta_{\nu_{p}}^{\mu_{p}}\right]$ is a totally antisymmetric product of $p$ Kronecker deltas.

Varying the action with respect to the metric, one obtains the equations of motion (EOM), which correspond to Einstein tensor plus fourth-order contributions coming from curvature-squared terms; namely

$$
\begin{aligned}
0= & \frac{1}{\kappa} G_{\mu \nu}+2 \beta R\left(R_{\mu \nu}-\frac{1}{4} g_{\mu \nu} R\right) \\
& +(2 \beta+\alpha)\left(g_{\mu \nu} \square-\nabla_{\mu} \nabla_{\nu}\right) R \\
& -\gamma H_{\mu \nu}+\alpha \square G_{\mu \nu}+2 \alpha\left(R_{\mu \sigma \nu \rho}-\frac{1}{4} g_{\mu \nu} R_{\sigma \rho}\right) R^{\sigma \rho},
\end{aligned}
$$

where the part that is linear in the curvature, namely,

$$
G_{\nu}^{\mu}=R_{\nu}^{\mu}-\frac{1}{2} R \delta_{\nu}^{\mu}+\Lambda_{0} \delta_{\nu}^{\mu}
$$

is the usual field equation of GR, and the quadratic part

$$
H_{\nu}^{\mu}=\frac{1}{8} \delta_{\left[\nu \nu_{1} \nu_{2} \nu_{3} \nu_{4}\right]}^{\left[\mu \mu_{1} \mu_{2} \mu_{3} \mu_{4}\right]} R_{\mu_{1} \mu_{2}}^{\nu_{1} \nu_{2}} R_{\mu_{3} \mu_{4}}^{\nu_{2} \nu_{4}}
$$

corresponds to the Lanczos tensor [4-6].

Vacuum states of the theory correspond to maximally symmetric spaces, which satisfy the constant curvature condition

$$
R_{\alpha \beta}^{\mu \nu}=-\frac{1}{\ell_{\mathrm{eff}}^{2}} \delta_{[\alpha \beta]}^{[\mu \nu]} .
$$

Here, $\ell_{\text {eff }}$ is the effective (A)dS radius. From the equation of motion one can readily obtain an expression for the effective (A)dS radius in terms of the couplings of the theory. Using the standard relation between (A)dS length and the corresponding cosmological constant $\Lambda_{\text {eff }}=$ $-(2 n-1)(2 n-2) / 2 \ell_{\mathrm{eff}}^{2}$, one arrives at the equation

$$
\begin{aligned}
-\frac{1}{2 \kappa \Lambda_{\mathrm{eff}}}+\frac{\Lambda_{0}}{2 \kappa \Lambda_{\mathrm{eff}}^{2}}= & \frac{(2 n-4)}{(2 n-2)^{2}}(2 n \beta+\alpha) \\
& +\gamma \frac{(2 n-4)(2 n-3)}{(2 n-2)(2 n-1)} .
\end{aligned}
$$

Note that $\Lambda_{\text {eff }}=\Lambda_{0}$ in four dimensions. This is no longer the case in $D>4$, as higher curvature terms modify the effective cosmological constant.

The surface term of the theory arises from integrating by parts the variation of the gravitational action in order to 
construct the EOMs. Without loss of generality, one can always split such contribution in the pieces that contain $\delta g$ and $\delta \Gamma$, respectively. They can be easily derived employing Wald construction of Noether conserved quantities in gravity theories [34]. Then, the surface term is cast in the form

$$
\Theta^{\alpha}(\delta g, \delta \Gamma)=2 E_{\mu \nu}^{\alpha \beta} g^{\nu \lambda} \delta \Gamma_{\beta \lambda}^{\mu}+2 \nabla^{\mu} E_{\mu \nu}^{\alpha \beta}\left(g^{-1} \delta g\right)_{\beta}^{\nu},
$$

where the tensor $E_{\mu \nu}^{\alpha \beta}=\delta \mathcal{L} / \delta R_{\alpha \beta}^{\mu \nu}$ is the derivative of the Lagrangian density with respect to the Riemann tensor which, for QCG theory, yields

$$
\begin{aligned}
E_{\mu \nu}^{\alpha \beta}= & \frac{1}{2 \kappa} \delta_{[\mu \nu]}^{[\alpha \beta]}+\frac{1}{2} \alpha R_{[\mu}^{[\alpha} \delta_{\nu]}^{\beta]}+\beta R \delta_{[\mu \nu]}^{[\alpha \beta]}+\frac{1}{2} \gamma \delta_{\left[\mu \nu \nu_{3} \nu_{4}\right]}^{\left[\alpha \beta \mu_{3} \mu_{4}\right]} R_{\mu_{3} \mu_{4}}^{\nu_{3} \nu_{4}} \\
& +\frac{n \alpha_{2 n}}{2^{n}} \delta_{\left[\mu \nu \nu_{3} \cdots \nu_{2 n}\right]}^{\left.\alpha \beta \mu_{2} \cdots \mu_{2 n}\right]} R_{\mu_{3} \mu_{4}}^{\nu_{3} \nu_{4}} \cdots R_{\mu_{2 n-1} \nu_{2 n}}^{\nu_{2 n} \nu_{2 n}} .
\end{aligned}
$$

In what follows, we construct an energy definition for QCG in even dimensions, as the natural generalization of the procedure shown in Ref. [33].

\section{ENERGY DEFINITION}

In the literature, there is a plethora of different approaches to deal with the general issue of defining energy for a given gravity theory [38]. Here, we shall apply Wald formalism $[34,35]$, as the charges derived with such formalism for the case of quadratic theories take a relatively simple form: For a Lagrangian which is a function of the metric and the Riemann curvature $\mathcal{L}\left(g_{\mu \nu}, R_{\mu \nu \alpha \beta}\right)$, the conserved quantity associated to any Killing vector $\xi^{\mu}$ is expressed as a surface integral in the codimension-2 surface $\Sigma$

$$
Q_{\mathrm{W}}^{\alpha}[\xi]=2 \int_{\Sigma} d S_{\beta}\left(E_{\mu \nu}^{\alpha \beta} \nabla^{\mu} \xi^{\nu}+2 \nabla^{\mu} E_{\mu \nu}^{\alpha \beta} \xi^{\nu}\right) .
$$

The use of Wald's derivation of conserved quantities from the gravitational bulk Lagrangian does not guarantee by itself that the value of the energy is correct. This can be seen in GR, where Wald charge coincides with the Komar formula. So, even in the asymptotically flat case, the black hole mass computed from (10) does not coincide with the one obtained by the Hamiltonian method. The situation worsens for AdS gravity, as the behavior of solutions turns Komar integral divergent at the spatial infinity. These facts imply that the charges (10) need to be corrected by adding suitable boundary terms to the original Lagrangian. Prescribing appropriate boundary conditions makes possible the integration of the conserved quantities, although getting closed expressions for the charges is not always guaranteed. In this regard, the covariant formalism [32], which provides a robust method to compute conserved charges in which the boundary terms are constructed systematically, is particularly useful. As we will see, we will obtain a result in agreement with that method.
The addition of topological terms to Einstein-Hilbert action in GR with a negative cosmological constant provides a remarkably simple method to circumvent the drawbacks in the procedure described above. In four dimensions, the consistent coupling of the Gauss-Bonnet and Pontryagin terms allows us to express the gravitational charges as the electric/magnetic part of the Weyl tensor $[39,40]$. The formulas, which give rise to the correct energy of different asymptotically AdS solutions, turn the discussions on background-substraction methods and the inclusion of extra boundary terms in the Lagrangian superfluous. In a previous work [33], we have shown that the addition of the Gauss-Bonnet invariant to quadraticcurvature gravity (QCG) action in $D=4$ also acts as a regulator of the Noether charges in both Einstein and nonEinstein sectors of the theory. Here, we extend this result to an arbitrary even-dimensional QCG theory. A single topological term added to the action suffices to render the conserved charges finite. The information about the background of the corresponding sector of the theory is encoded in the asymptotic value of the curvature, and gets reflected in the coupling constant of the Euler topological term. In other words, even in the higher-derivative theory it happens that the addition of topological invariants to the action is sufficient to regularize the conserved charges, making the job of the otherwise needed boundary terms.

In QCG, due to Bianchi identity, the second part of the integrand in Eq. (10) does not feature any term containing $\gamma$. Altogether, the rest of $\nabla^{\mu} E_{\mu \nu}^{\alpha \beta}$ vanishes identically for Einstein spaces,

$$
R_{\mu \nu}=-\frac{(2 n-1) g_{\mu \nu}}{\ell_{\mathrm{eff}}^{2}} .
$$

The gravity theory under analysis here admits a number of analytic solutions with different asymptotic behavior, such as asymptotically AdS, Lifshitz, etc. These different asymptotic behaviors represent different sectors of the theory. For the purpose of the present discussion, we will consider solutions which are continuously connected to a global AdS spacetime, which defines the background configuration. We then fix the coupling of the Euler density in terms of the parameters $\alpha, \beta$, and $\gamma$ by the following criterion: The total surface term must vanish identically for the vacuum state (6) corresponding to the class of solutions we are interested in. This basic assumption implies a well-posed variational principle, at least for global AdS spacetime. In doing so, the coupling constant of the Euler density reads

$$
\begin{aligned}
\alpha_{2 n}= & \frac{(-1)^{n} \ell_{\mathrm{eff}}^{2 n-2}}{n(2 n-2) ! \kappa}\left[1+\frac{4 \kappa \Lambda_{\mathrm{eff}}}{2 n-2}(2 n \beta+\alpha\right. \\
& \left.\left.+\gamma \frac{(2 n-3)(2 n-2)}{2 n-1}\right)\right]
\end{aligned}
$$


The quartic relation for the effective AdS radius in (7) has a single root provided the derivative of it with respect to $\ell_{\mathrm{eff}}^{2}$ is different from zero. Indeed, this degeneracy condition poses an obstruction for the linearization of EOM when the two maximally symmetric vacua of the theory coincide. This is similar to what happens, e.g., in odd dimensions with the Chern-Simons gravity theories [41]. The construction of conserved quantities is sensitive to this issue, as linearized charges cannot be obtained for the degenerate case. In relation to this, it is worth emphasizing that the method of adding the Euler term (12) is not affected by this consideration, as it does not rely on linear perturbations of the geometry around a given background. Once we have suitably identified the vacuum solution, we can ask whether the massive deviations from the maximally symmetric solution are such that the total surface integral keeps being finite. This can be checked in concrete examples.

The full charge is obtained as the surface integral

$$
Q^{\alpha}[\xi]=\int_{\Sigma} d S_{\beta}\left(q_{(1)}^{\alpha \beta}+q_{(2)}^{\alpha \beta}\right),
$$

where $q_{(1)}^{\alpha \beta}$ and $q_{(2)}^{\alpha \beta}$ are the prepotentials

$$
\begin{aligned}
q_{(1)}^{\alpha \beta}= & \nabla^{\mu} \xi^{\nu}\left(\frac{1}{\kappa} \delta_{[\mu \nu]}^{[\alpha \beta]}+\alpha R_{[\mu}^{[\alpha} \delta_{\nu]}^{\beta]}+2 \beta R \delta_{[\mu \nu]}^{[\alpha \beta]}+\gamma \delta_{\left[\mu \nu \nu_{3} \nu_{4}\right]}^{\left[\alpha \beta \mu_{3} \mu_{4}\right]} R_{\mu_{3} \mu_{4}}^{\nu_{3} \nu_{4}}\right. \\
& \left.+\frac{n \alpha_{2 n}}{2^{n-1}} \delta_{\left[\mu \nu \cdots \nu_{2 n}\right]}^{\left[\alpha \beta \cdots \mu_{2 n}\right]} R_{\mu_{3} \mu_{4}}^{\nu_{3} \nu_{4}} \cdots R_{\mu_{2_{n-1}} \nu_{2_{n}}}^{\nu_{2 n-1} \nu_{2 n}}\right),
\end{aligned}
$$

and

$$
q_{(2)}^{\alpha \beta}=2 \nabla^{\mu}\left(\alpha R_{[\mu}^{[\alpha} \delta_{\nu]}^{\beta]}+2 \beta R \delta_{[\mu \nu]}^{[\alpha \beta]}\right) \xi^{\nu} .
$$

Now, we can consider a static black hole with standard AdS asymptotics, whose metric is given by

$$
d s^{2}=-f^{2}(r) d t^{2}+\frac{1}{f^{2}(r)} d r^{2}+r^{2} d \Omega_{2 n-2}^{2},
$$

where $d \Omega_{2 n-2}^{2}$ is the metric on the unit $(2 n-2)$-sphere. This discussion can be straightforwardly generalized to topological black holes with planar or hyperbolic horizons. The nonvanishing components of the Riemann curvature for this static configuration are

$$
\begin{aligned}
R_{t r}^{t r} & =-\frac{1}{2}\left(f^{2}\right)^{\prime \prime}, \\
R_{t m}^{t n} & =R_{r m}^{r n}=-\frac{1}{2 r}\left(f^{2}\right)^{\prime} \delta_{m}^{n}, \\
R_{k l}^{m n} & =\frac{1-f^{2}}{r^{2}} \delta_{[k l]}^{[m n]},
\end{aligned}
$$

while the nonvanishing components of the Ricci tensor are

$$
\begin{aligned}
R_{t}^{t} & =R_{r}^{r}=-\frac{1}{2 r}\left[r\left(f^{2}\right)^{\prime \prime}+2(n-1)\left(f^{2}\right)^{\prime}\right], \\
R_{m}^{n} & =-\frac{1}{r^{2}}\left[r\left(f^{2}\right)^{\prime}-(2 n-3)\left(1-f^{2}\right)\right] \delta_{m}^{n} .
\end{aligned}
$$

Here, the prime stands for derivatives with respect to $r$. In the case of Boulware-Deser black holes [8] of EinsteinGauss-Bonnet gravity $(\alpha=\beta=0)$, which is the working example in QCG, the metric function $f^{2}(r)$ takes the asymptotic form

$$
f^{2}(r) \simeq \frac{r^{2}}{\ell_{\mathrm{eff}}^{2}}+1-\left(\frac{r_{0}}{r}\right)^{2 n-3}+\cdots,
$$

with $\ell_{\mathrm{eff}}^{2}$ being a root of the polynomial equation

$$
\gamma(2 n-3)(2 n-4) \frac{1}{\ell_{\mathrm{eff}}^{4}}-\frac{1}{\kappa \ell_{\mathrm{eff}}^{2}}+\frac{1}{\kappa \ell^{2}}=0 .
$$

The ellipsis in (18) stands for higher powers of $1 / r$. In a more general case, in which the couplings $\alpha$ and $\beta$ are nonzero and generic, a similar falloff is expected, with the difference being the value of the AdS radius, which turns out to be given by Eq. (7). For special relations between $\alpha$, $\beta$, and $\Lambda_{0}$, a weakened version of the asymptotic condition (18) might be possible. This has been extensively studied in the literature. However, for generic values of the parameters $\alpha$ and $\beta$, (18) is the expected behavior, as the quadratic terms represent ultraviolet corrections. A more general case could eventually involve two different metric functions corresponding to $g_{t t}$ and $g_{r r}^{-1}$, each one with the asymptotic behavior that respects the AdS asymptotics as given by Eq. (18), what we discuss at the end of this section.

For static massive objects, the energy formula takes the form

$$
\begin{aligned}
E= & Q^{t}\left[\partial_{t}\right] \\
= & \int_{\Sigma} d S_{r} \nabla^{r} \xi^{t}\left[\alpha\left(R_{t}^{t}+R_{r}^{r}\right)+\left(\frac{1}{\kappa}+2 \beta R\right) \delta_{[r t]}^{[r t]}\right. \\
& \left.+\gamma \delta_{\left[r t m_{1} m_{2}\right]}^{\left.r t p_{1} p_{2}\right]} R_{p_{1} p_{2}}^{m_{1} m_{2}}+\frac{n \alpha_{2 n}}{2^{n-1}} \delta_{\left[r t m_{1} \cdots m_{2 n}\right]}^{\left[r t p_{1} \cdots p_{2 n}\right]} R_{p_{1} p_{2}}^{m_{1} m_{2}} \cdots R_{p_{2 n-3} p_{2 n-2}}^{m_{2 n-3} m_{2 n-2}}\right] .
\end{aligned}
$$

Given the form of the line element for the black hole (15), explicit evaluation of the above energy also requires the derivative of the timelike Killing vector, $\nabla^{r} \xi^{t}=\left(f^{2}\right)^{\prime} / 2$, and the codimension-2 surface element $d S_{r}$. If the local coordinates on $\Sigma$ are denoted by $y^{m}$ and the corresponding line element of $\Sigma$ is

$$
r^{2} d \Omega_{2 n-2}^{2}=r^{2} \sigma_{m n}(y) d y^{m} d y^{n},
$$

where $\sigma_{m n}$ is the metric of the unit sphere, then we find along the radial normal $d S_{r}=r^{2 n-2} d^{2 n-2} y \sqrt{\sigma}$. In turn, the 
curvature expression in the square bracket can be recast in terms of the metric function $f^{2}(r)$ and its derivatives. The result is

$$
\begin{aligned}
E= & \int_{\Sigma} d^{2 n-2} y \sqrt{\sigma} \frac{1}{2}\left(f^{2}\right)^{\prime}\left\{\frac{1}{\kappa}-\frac{\alpha}{r}\left(r\left(f^{2}\right)^{\prime \prime}+2(n-1)\left(f^{2}\right)^{\prime}\right)\right. \\
& -\frac{2 \beta}{r^{2}}\left[r^{2}\left(f^{2}\right)^{\prime \prime}+4(n-1) r\left(f^{2}\right)^{\prime}\right. \\
& \left.-2(n-1)(2 n-3)\left(1-f^{2}\right)\right] \\
& +4 \gamma(n-1)(2 n-3) \frac{1-f^{2}}{r^{2}} \\
& \left.+n \alpha_{2 n}(2 n-2) !\left(\frac{1-f^{2}}{r^{2}}\right)^{n-1}\right\} .
\end{aligned}
$$

It is important to notice here that the Ricci tensor tends to a constant value, namely $R_{\nu}^{\mu} \simeq-(2 n-1) \delta_{\nu}^{\mu} / \ell_{\text {eff }}^{2}$.

Replacing the asymptotic value of the metric function and its derivatives and performing the integral on the angular variables $\int_{\Sigma} d^{2 n-2} y \sqrt{\sigma}=\operatorname{Vol}\left(S^{2 n-2}\right)$, the expression for the energy takes the form

$$
\begin{aligned}
E= & \operatorname{Vol}\left(S^{2 n-2}\right) \lim _{r \rightarrow \infty} r^{2 n-2} f f^{\prime}\left[\frac{1}{\kappa}-(\alpha+2 \beta)\left(f^{2}\right)^{\prime \prime}\right. \\
& -2(n-1)(\alpha+4 \beta) \frac{\left(f^{2}\right)^{\prime}}{r} \\
& +4(n-1)(2 n-3) \frac{1-f^{2}}{r^{2}}(\beta+\gamma) \\
& \left.+n \alpha_{2 n}(2 n-2) !\left(\frac{1-f^{2}}{r^{2}}\right)^{n-1}\right]
\end{aligned}
$$

where the last term of this expression can be expanded as

$$
\left(\frac{1-f^{2}}{r^{2}}\right)^{n-1}=\frac{(-1)^{n-1}}{\ell_{\mathrm{eff}}^{2 n-2}}+(n-1) \frac{(-1)^{n-2}}{\ell_{\mathrm{eff}}^{2 n-4}} \frac{r_{0}^{2 n-3}}{r^{2 n-1}}+\cdots
$$

The remaining terms in the sum can be neglected as they vanish in the limit $r \rightarrow \infty$, in which the surface integral is evaluated. Keeping all the coupling constants arbitrary, we obtain the divergent expression

$$
\begin{aligned}
E= & \operatorname{Vol}\left(S^{2 n-2}\right) \lim _{r \rightarrow \infty}\left[C\left(\frac{r^{2 n-1}}{\ell_{\mathrm{eff}}^{2}}+\frac{(2 n-3) r_{0}^{2 n-3}}{2}\right)\right. \\
& \left.+\left(2(2 n-3) \gamma+n \alpha_{2 n}(2 n-2) ! \frac{(-1)^{n-2}}{2 \ell_{\mathrm{eff}}^{2 n-4}}\right)\right] \\
& \times \frac{(2 n-2) r_{0}^{2 n-3}}{\ell_{\mathrm{eff}}^{2}},
\end{aligned}
$$

where we introduced the constant

$$
\begin{aligned}
C= & \frac{1}{\kappa}-\frac{2}{\ell_{\mathrm{eff}}^{2}}((2 n-1)(\alpha+2 n \beta)+(2 n-2)(2 n-3) \gamma) \\
& +n \alpha_{2 n}(2 n-2) ! \frac{(-1)^{n-1}}{\ell_{\mathrm{eff}}^{2 n-2}} .
\end{aligned}
$$

Now it is clear that fixing the value of $\alpha_{2 n}$ as in Eq. (12), which produces $C=0$ and regularizes the $r^{2 n-1}$ term, makes the gravitational energy finite.

Finally, writing $\gamma$ in terms of $\alpha$ and $\beta$ [as given in Eq. (7)], the energy is

$$
E=\left(-1+\frac{8 \Lambda_{\mathrm{eff}} \kappa}{(2 n-2)^{2}}(2 n \beta+\alpha)+\frac{2 \Lambda_{0}}{\Lambda_{\mathrm{eff}}}\right) \frac{(2 n-2) r_{0}^{2 n-3}}{4 G_{2 n}},
$$

which is the correct result for a black hole mass in QCG theory with $\Lambda_{0}=0$, cf. [27], in our case generalized to include the bare cosmological constant $\Lambda_{0}$.

In the case when the metric component $g_{t t}$ in the ansatz is replaced by a more general function $-N^{2}(r) f^{2}(r)$ that respects the AdS asymptotics, the resulting formula for the energy still yields a finite result. Here, in order to compare with known results in the literature, we consider the case $N=1$.

The above discussion shows the effect of the Euler topological invariant added to the QCG action, which is similar to the one in GR and Einstein-Gauss-Bonnet gravity actions. Namely, without such term, the gravitational energy is both divergent and yields an incorrect value for the finite part in the mass computation. The addition of properly fixed Euler coupling $\alpha_{2 n}$ resolves both issues at the same time.

\section{CONCLUSIONS}

In this paper, we derived a general formula for the conserved charges for asymptotically AdS black holes in quadratic curvature gravity in $2 n$ dimensions. The addition of topological terms to the gravity action acts as a regulator at the level of the surface term, and yields finite Wald charges in AdS without need of extra boundary terms. It remains an open question to know whether the method of adding Euler characteristics can also be implemented in order to compute conserved charges in sectors with other asymptotic behaviors, such Lifshitz black holes, AdSwaves, or spacetimes with weakened asymptotics in AdS.

In the past, the use of topological terms to regularize the conserved quantities and the Euclidean action of a given gravity theory has not only provided a powerful, yet simple computational tool to understand physical properties of black holes, but it has also unveiled remarkable features of the gravitational action in critical points of the parameter space in four dimensions such as critical gravity [14]. Indeed, the addition of the Gauss-Bonnet term to the critical gravity action makes manifest the fact that the energy, the entropy, and the action are zero for Einstein spaces [42,43]. 
Implications of this procedure in the computation of holographic correlation functions have been explored in Ref. [44]. We expect that the addition of Euler terms will make more clear certain properties of critical theories in higher dimensions too.

\section{ACKNOWLEDGMENTS}

This work was funded in part by the NSF through Grant No. PHY-1214302, Chilean FONDECYT Grants
No. 1170765 and No. 1190533, and the Grant VRIEAPUCV No. 123.764/2019. D. R. B. is supported by Becas Chile (CONICYT) Ph.D. scholarship No. 72200301. G. G. is partially supported by CONICET through Grant No. PIP 1109 (2017).

Note added.-While finishing our paper, the work [45] appeared, in which the higher-dimensional extension of [33] is also worked out.
[1] D. J. Gross and E. Witten, Superstring modifications of Einstein's equations, Nucl. Phys. B277, 1 (1986).

[2] R. R. Metsaev and A. A. Tseytlin, Order alpha-prime (two loop) equivalence of the string equations of motion and the sigma model Weyl invariance conditions: Dependence on the dilaton and the antisymmetric tensor, Nucl. Phys. B293, 385 (1987).

[3] B. Zwiebach, Curvature squared terms and string theories, Phys. Lett. B 156, 315 (1985).

[4] C. Lanczos, A remarkable property of the RiemannChristoffel tensor in four dimensions, Ann. Math. 39, 842 (1938).

[5] D. Lovelock, The Einstein tensor and its generalizations, J. Math. Phys. (N.Y.) 12, 498 (1971).

[6] D. Lovelock, The four-dimensionality of space and the Einstein tensor, J. Math. Phys. (N.Y.) 13, 874 (1972).

[7] A. A. Starobinsky, A new type of isotropic cosmological models without singularity, Phys. Lett. B 91, 99 (1980); Adv. Ser. Astrophys. Cosmol. 3, 130 (1987).

[8] D. G. Boulware and S. Deser, String Generated Gravity Models, Phys. Rev. Lett. 55, 2656 (1985).

[9] K. S. Stelle, Classical gravity with higher derivatives, Gen. Relativ. Gravit. 9, 353 (1978).

[10] S. Ferrara and B. Zumino, Structure of conformal supergravity, Nucl. Phys. B134, 301 (1978).

[11] K. S. Stelle, Renormalization of higher derivative quantum gravity, Phys. Rev. D 16, 953 (1977).

[12] A. Gruzinov and M. Kleban, Causality constrains higher curvature corrections to gravity, Classical Quantum Gravity 24, 3521 (2007).

[13] X. O. Camanho, J. D. Edelstein, J. Maldacena, and A. Zhiboedov, Causality constraints on corrections to the graviton three-point coupling, J. High Energy Phys. 02 (2016) 020.

[14] H. Lu and C. N. Pope, Critical Gravity in Four Dimensions, Phys. Rev. Lett. 106, 181302 (2011).

[15] S. Deser, H. Liu, H. Lu, C. N. Pope, T. C. Sisman, and B. Tekin, Critical points of $D$-dimensional extended gravities, Phys. Rev. D 83, 061502 (2011).

[16] W. Li, W. Song, and A. Strominger, Chiral gravity in three dimensions, J. High Energy Phys. 04 (2008) 082.
[17] J. Maldacena, Einstein gravity from conformal gravity, arXiv:1105.5632.

[18] J. Oliva and S. Ray, Classification of six derivative Lagrangians of gravity and static spherically symmetric solutions, Phys. Rev. D 82, 124030 (2010).

[19] J. Oliva and S. Ray, A new cubic theory of gravity in five dimensions: Black hole, Birkhoff's theorem and C-function, Classical Quantum Gravity 27, 225002 (2010).

[20] R. C. Myers and B. Robinson, Black holes in quasitopological gravity, J. High Energy Phys. 08 (2010) 067.

[21] R. A. Hennigar, D. Kubizk, and R. B. Mann, Generalized quasitopological gravity, Phys. Rev. D 95, 104042 (2017).

[22] P. Bueno and P. A. Cano, On black holes in higherderivative gravities, Classical Quantum Gravity 34, 175008 (2017).

[23] P. Bueno, P. A. Cano, and R. A. Hennigar, (Generalized) quasi-topological gravities at all orders, Classical Quantum Gravity 37, 015002 (2020).

[24] P. Bueno and P. A. Cano, Einsteinian cubic gravity, Phys. Rev. D 94, 104005 (2016).

[25] G. Arciniega, J. D. Edelstein, and L. G. Jaime, Towards geometric inflation: The cubic case, arXiv:1810.08166.

[26] S. Deser and B. Tekin, Gravitational Energy in Quadratic Curvature Gravities, Phys. Rev. Lett. 89, 101101 (2002).

[27] S. Deser and B. Tekin, Energy in generic higher curvature gravity theories, Phys. Rev. D 67, 084009 (2003).

[28] L. F. Abbott and S. Deser, Stability of gravity with a cosmological constant, Nucl. Phys. B195, 76 (1982).

[29] N. Okuyama and J. Koga, Asymptotically anti de Sitter spacetimes and conserved quantities in higher curvature gravitational theories, Phys. Rev. D 71, 084009 (2005).

[30] Y. Pang, Brief note on Ashetkar-Magnon-Das conserved quantities in quadratic curvature theories, Phys. Rev. D 83, 087501 (2011).

[31] T. Azeyanagi, G. Compere, N. Ogawa, Y. Tachikawa, and S. Terashima, Higher-derivative corrections to the asymptotic virasoro symmetry of $4 \mathrm{~d}$ extremal black holes, Prog. Theor. Phys. 122, 355 (2009).

[32] G. Barnich and F. Brandt, Covariant theory of asymptotic symmetries, conservation laws and central charges, Nucl. Phys. B633, 3 (2002). 
[33] G. Giribet, O. Miskovic, R. Olea, and D. Rivera-Betancour, Energy in higher derivative gravity via topological regularization, Phys. Rev. D 98, 044046 (2018).

[34] V. Iyer and R. M. Wald, Some properties of Noether charge and a proposal for dynamical black hole entropy, Phys. Rev. D 50, 846 (1994).

[35] R. M. Wald and A. Zoupas, General definition of conserved quantities in general relativity and other theories of gravity, Phys. Rev. D 61, 084027 (2000).

[36] R. Aros, M. Contreras, R. Olea, R. Troncoso, and J. Zanelli, Conserved Charges for Gravity with Locally AdS Asymptotics, Phys. Rev. Lett. 84, 1647 (2000).

[37] R. Aros, M. Contreras, R. Olea, R. Troncoso, and J. Zanelli, Conserved charges for even dimensional asymptotically AdS gravity theories, Phys. Rev. D 62, 044002 (2000).

[38] H. Adami, M. R. Setare, T. C. Sisman, and B. Tekin, Conserved charges in extended theories of gravity, Phys. Rep. 834, 1 (2019).
[39] O. Miskovic and R. Olea, Topological regularization and self-duality in four-dimensional anti-de Sitter gravity, Phys. Rev. D 79, 124020 (2009).

[40] R. Araneda, R. Aros, O. Miskovic, and R. Olea, Magnetic mass in 4D AdS gravity, Phys. Rev. D 93, 084022 (2016).

[41] G. Arenas-Henriquez, O. Miskovic, and R. Olea, Vacuum degeneracy and conformal mass in Lovelock AdS gravity, J. High Energy Phys. 11 (2017) 128.

[42] O. Miskovic, R. Olea, and M. Tsoukalas, Renormalized AdS action and critical gravity, J. High Energy Phys. 08 (2014) 108.

[43] G. Anastasiou, R. Olea, and D. Rivera-Betancour, NoetherWald energy in critical gravity, Phys. Lett. B 788, 302 (2019).

[44] G. Anastasiou and R. Olea, Holographic correlation functions in critical gravity, J. High Energy Phys. 11 (2017) 019.

[45] M.-L. Wang and J.-J. Peng, A note on the definition of gravitational energy for quadratic curvature gravity via topological regularization, arXiv:2001.07996. 